\title{
CLOPIDOGREL AND TICAGRELOR DO NOT INFLUENCE PLATELET INDICES AFTER PERCUTANEOUS CORONARY INTERVENTION
}

\author{
IOANA ȘUȘ ${ }^{1}$, LASZLO HADADI ${ }^{2}$, CRISTINA SOMKEREKI ${ }^{3}$, DAN DOBREANU ${ }^{4}$ \\ 1,3,4 “George Emil Palade” University of Medicine, Pharmacy, Science and Technology of Târgu-Mureş, ${ }^{1,2,4}$ Emergency Institute for Cardiovascular \\ Diseases and Transplantation, Târgu-Mureş
}

\begin{abstract}
Keywords: percutaneous Abstract: Platelet indices have been linked to the severity and prognosis of coronary artery disease, coronary intervention, but a very small number of studies assessed them under dual antiplatelet therapy after percutaneous platelets, mean platelet coronary intervention (PCI). The aim of this study was to evaluate changes in mean platelet volume volume, antiplatelet $(M P V)$, platelet distribution width $(P D W)$ and platelet-large cell ratio $(P-L C R)$ in these patients. A therapy number of 437 patients with stable coronary artery disease, 131 patients with non ST segment elevation acute coronary syndrome and 151 patients with ST segment elevation myocardial infarction were included in the study. There was no difference between the three groups regarding platelet indices prior to PCI. Follow-up data was available for 181 patients, at a mean follow-up time of 69 (46-98) days. MPV, PDW and PLC-R were similar to those prior to PCI in all three groups. Regarding the P2Y12 inhibitor, clopidogrel or ticagrelor, there was no difference at follow-up between platelet indices irrespective of the indication for PCI.
\end{abstract}

\section{INTRODUCTION}

Platelets have been extensively studied as important factors in the occurrence of acute coronary syndromes. Mean platelet volume has been inversely correlated with the number of platelets.(1) Also, younger or activated platelets have a higher MPV and this has been linked to the severity $(2,3)$ and prognosis $(4,5)$ of coronary artery disease (CAD). As patients with CAD undergoing percutaneous coronary intervention with stent implantation have an indication for long-term dual antiplatelet therapy including aspirin and a P2Y12 inhibitor, we hypothesised that this could influence platelets size both due to platelet inhibition and changes in platelet turnover.

\section{AIM}

The aim of this study was to evaluate changes in easily-accessible platelet indices such as mean platelet volume (MPV), platelet distribution width (PDW) and platelet-large cell ratio (P-LCR) under dual antiplatelet therapy in patients undergoing PCI.

\section{MATERIALS AND METHODS}

A retrospective observational study was performed including consecutive patients undergoing coronary angioplasty at the Emergency Institute for Cardiovascular Disease and Transplantation of Târgu-Mureş in January-December 2016. Data were collected from Percutaneous Coronary Intervention registry for patient and procedural data and laboratory data were extracted from patients' electronic files. Patients with thrombolysis, acute infections and hematologic disorders were excluded.

History of diabetes mellitus, arterial hypertension and dyslipidemia were considered if these diagnoses were mentioned in medical records or patient had specific treatment. Patients were further divided into three groups based on the indication for PCI: stable coronary artery disease (SCAD), non-ST segment elevation acute coronary syndrome (NSTE-ACS) and ST segment elevation myocardial infarction (STEMI). Every group was further divided into two subgroups based on the antiplatelet drug chosen in association to aspirin after PCI.

The first blood laboratory measurements performed on admission were recorded. Hemogram and standard biochemistry data were included in the analysis. Platelet indices, MPV, PDW and P-LCR were measured by automated hematological analyser Sysmex Xs-800i and calculated using standardized formulas. Follow-up laboratory data was available for patients who were subsequently hospitalized in our department for investigations, so laboratory tests prior and after PCI were performed in the same laboratory allowing for comparable data.

Data was statistically analysed using MedCalc Statistical Software version 17.9.7 (MedCalc Software bvba, Ostend, Belgium).

\section{RESULTS}

A number of 437 patients with SCAD, 131 patients with NSTE-ACS and 151 patients with STEMI were included in the study. The patients in the three groups had similar ages, but the gender distribution was different with a higher female: male ratio in the STEMI group. Also, patients in the STEMI group were more frequently smokers and had a history of arterial hypertension (table no. 1).

Prior to PCI, WBC and neutrophils count were assessed. Although previously diagnosed dyslipidemia was most frequent in the SCAD group, total cholesterol and LDLcholesterol levels were significantly higher at presentation in the STEMI group significantly higher and lymphocyte count was lower in patients presenting with STEMI $(\mathrm{p}<0.05)$.

${ }^{1}$ Corresponding author: Ioana Şuş, Str. Gh. Marinescu, Nr. 50, Tîrgu-Mureş, România, E-mail susioana@yahoo.com, Phone: +40740970553 Article received on 27.06.2020 and accepted for publication on 02.12.2020 
CLINICAL ASPECTS

Table no. 1. Baseline patient data

\begin{tabular}{|l|c|c|c|c|}
\hline & $\begin{array}{c}\text { SCAD } \\
\mathbf{n = 4 3 7}\end{array}$ & $\begin{array}{c}\text { NSTE-ACS } \\
\mathbf{n = 1 3 1}\end{array}$ & $\begin{array}{c}\text { STEMI } \\
\mathbf{N = 1 5 1}\end{array}$ & $\mathbf{p}$ \\
\hline Age (years) & $\begin{array}{c}64 \\
(56-70)\end{array}$ & $\begin{array}{c}65 \\
(53-71)\end{array}$ & $\begin{array}{c}63 \\
(53-71))\end{array}$ & 0.35 \\
\hline $\begin{array}{l}\text { Female } \\
\text { gender }\end{array}$ & $\begin{array}{c}105 \\
(24 \%)\end{array}$ & $\begin{array}{c}47 \\
(35.8 \%)\end{array}$ & $\begin{array}{c}51 \\
(33.8 \%)\end{array}$ & $\mathbf{0 . 0 7}$ \\
\hline Smoking & $\begin{array}{c}251 \\
(57.4 \%)\end{array}$ & $\begin{array}{c}74 \\
(56.4 \%)\end{array}$ & $\begin{array}{c}100 \\
(66.2 \%)\end{array}$ & $<\mathbf{0 . 0 1}$ \\
\hline $\begin{array}{l}\text { Arterial } \\
\text { hypertension }\end{array}$ & $\begin{array}{c}368 \\
(85 \%)\end{array}$ & $\begin{array}{c}98 \\
(79 \%)\end{array}$ & $\begin{array}{c}98 \\
(66.2 \%)\end{array}$ & $<\mathbf{0 . 0 1}$ \\
\hline $\begin{array}{l}\text { History of } \\
\text { MI }\end{array}$ & $\begin{array}{c}162 \\
(37.2 \%)\end{array}$ & $\begin{array}{c}33 \\
(25.2 \%)\end{array}$ & $\begin{array}{c}24 \\
(15.9 \%)\end{array}$ & $<\mathbf{0 . 0 1}$ \\
\hline $\begin{array}{l}\text { History of } \\
\text { dyslipidemia }\end{array}$ & $\begin{array}{c}274 \\
(62.8 \%)\end{array}$ & $\begin{array}{c}78 \\
(60.0 \%)\end{array}$ & $\begin{array}{c}39 \\
(25.8 \%)\end{array}$ & $<\mathbf{0 . 0 1}$ \\
\hline $\begin{array}{l}\text { Three-vessel } \\
\text { disease }\end{array}$ & $\begin{array}{c}161 \\
(37.9 \%)\end{array}$ & $\begin{array}{c}51 \\
(39.2 \%)\end{array}$ & $\begin{array}{c}50 \\
(33.6 \%)\end{array}$ & 0.58 \\
\hline
\end{tabular}

Regarding platelets, there was no difference between the three groups neither regarding their number, nor standard platelet indices (table no. 2).

Table no. 2. Baseline hemogram parameters in patients undergoing percutaneous coronary

\begin{tabular}{|c|c|c|c|c|}
\hline & $\begin{array}{l}\text { SCAD } \\
\mathbf{n}=\mathbf{4 3 7}\end{array}$ & $\begin{array}{c}\text { NSTE-ACS } \\
\mathbf{n}=131\end{array}$ & $\begin{array}{l}\text { STEMI } \\
\mathbf{N}=151\end{array}$ & $\mathbf{P}$ \\
\hline $\mathrm{Hb}(\mathrm{g} / \mathrm{dl})$ & $\begin{array}{c}435 / 437 \\
13.8 \\
(12.7-14.7)\end{array}$ & $\begin{array}{c}130 / 131 \\
13.4 \\
(12.2-14.5)\end{array}$ & $\begin{array}{c}148 / 151 \\
13.1 \\
(11.8-14.3)\end{array}$ & 0.01 \\
\hline $\begin{array}{l}\text { WBC } \\
(/ \mathrm{dl})\end{array}$ & $\begin{array}{c}435 / 437 \\
7410 \\
(6140-8630)\end{array}$ & $\begin{array}{c}130 / 131 \\
7275 \\
(6090-8800)\end{array}$ & $\begin{array}{c}148 / 151 \\
9040 \\
(7570-10885)\end{array}$ & 0.01 \\
\hline $\begin{array}{c}\text { Neutroph } \\
\text { ils (/dl) }\end{array}$ & $\begin{array}{c}434 / 437 \\
4380 \\
(3460-5450)\end{array}$ & $\begin{array}{c}129 / 131 \\
4340 \\
(3527-5495) \\
\end{array}$ & $\begin{array}{c}148 / 151 \\
6120 \\
(4900-7730) \\
\end{array}$ & 0.01 \\
\hline $\begin{array}{l}\text { Lymphoc } \\
\text { ytes (/dl) }\end{array}$ & $\begin{array}{c}434 / 437 \\
1970 \\
(1510-2520)\end{array}$ & $\begin{array}{c}129 / 131 \\
1850 \\
(1505-2440) \\
\end{array}$ & $\begin{array}{c}148 / 151 \\
1690 \\
(1225-2380) \\
\end{array}$ & 0.02 \\
\hline $\begin{array}{c}\text { Platelet } \\
\text { count } \\
\left(* 10^{3} / \mu 1\right)\end{array}$ & $\begin{array}{c}435 / 437 \\
220 \\
(182.25-254)\end{array}$ & $\begin{array}{c}129 / 131 \\
222 \\
(179-261.25)\end{array}$ & $\begin{array}{c}146 / 148 \\
220 \\
(179-260)\end{array}$ & 0.93 \\
\hline $\begin{array}{c}\text { MPV } \\
(\mathrm{fL})\end{array}$ & $\begin{array}{c}435 / 437 \\
10.9 \\
(10.2-11.5)\end{array}$ & $\begin{array}{c}130 / 131 \\
10.8 \\
(10.3-11.6)\end{array}$ & $\begin{array}{c}148 / 151 \\
10.9 \\
(10.15-11.75)\end{array}$ & 0.56 \\
\hline $\begin{array}{c}\text { P-LCR } \\
(\%)\end{array}$ & $\begin{array}{c}435 / 437 \\
32.4 \\
(26.9-37.3) \\
\end{array}$ & $\begin{array}{c}130 / 131 \\
32.5 \\
(27.9-38.0)\end{array}$ & $\begin{array}{c}148 / 151 \\
32.4 \\
(26.2-39.8)\end{array}$ & 0.58 \\
\hline $\begin{array}{l}\text { PDW } \\
\text { (fL) }\end{array}$ & $\begin{array}{c}435 / 437 \\
13.0 \\
(11.8-14.6)\end{array}$ & $\begin{array}{c}130 / 131 \\
13.2 \\
(11.9-15.0)\end{array}$ & $\begin{array}{c}148 / 151 \\
13.1 \\
(11.8-15.2)\end{array}$ & 0.66 \\
\hline
\end{tabular}

Follow-up data was available for 181 patients, at a mean follow-up time of 69 (46-98) days. The MPV, PDW and PLC-R were similar to those prior to PCI in all three groups.
Concerning antiplatelet treatment, in patients with SCAD and NSTE-ACS, the majority of patients were on clopidogrel, but in the STEMI group an equal number of patients were on clopidogrel and ticagrelor respectively. Regarding the P2Y12 inhibitor, there was no difference at follow-up between platelet indices irrespective of the indication for PCI (table no. 3 ).

\section{DISCUSSIONS}

In patients undergoing percutaneous coronary intervention, dual antiplatelet therapy is compulsory in order to prevent coronary stent thrombosis. Nevertheless, a variable percentage of patients has a suboptimal response to antiaggregant treatment. Several hypotheses have tried to explain the high on-treatment platelet reactivity, among which increased platelet turnover.(6) One of the markers of platelet turnover is increased platelet volume, as young reticulated platelets released from the bone marrow have higher volumes. Also, activated platelets have higher volumes.(7) Still, conflicting data has been published until now.(8-10)

After short-term treatment with aspirin and clopidogrel, De Luca et al. observed a paradoxical increase in MPV in patients with acute coronary syndromes (1), which could be explained in the context of the acute event and stent implantation rather than due to treatment, but might be also caused by increase in platelet turnover. In our study, we evaluated platelet volume and derived indices long after the acute coronary syndrome to exclude the confounding factor of the acute event and we observed no increase in platelet count or indices under dual antiplatelet therapy, aspirin and clopidogrel or aspirin and ticagrelor, even if these are regimens with different proven potency.

All our patients, with SCAD or different types of acute coronary syndromes had stable PDW, MPV and P-LCR over time, showing that there was no increase in young platelet production irrespective of the antiplatelet drug used in addition to aspirin. This shows no difference in response to clopidogrelor or ticagrelor from the platelet morphological point of view. Of course, assessing reticulated platelets would have been a more reliable marker, so this is the main limitation of our study. Surprisingly, there was no difference in platelet indices when we compared the three indications for PCI, even if previous studies have shown that MPV is higher in patients with acute coronary syndromes.(11)

To our knowledge, there is no published study comparing platelet indices variability after long term treatment clopidogrel or ticagrelor.

Table no. 3. Follow-up hemogram parameters in patients undergoing percutaneous coronary intervention

\begin{tabular}{|c|c|c|c|c|c|c|c|c|c|}
\hline & \multicolumn{3}{|c|}{ SCAD } & \multicolumn{3}{|c|}{ NSTE-ACS } & \multicolumn{3}{|c|}{ STEMI } \\
\hline & $\begin{array}{l}\text { Clopidogrel } \\
\mathrm{N}=96\end{array}$ & $\begin{array}{c}\text { Ticagrelor } \\
\mathrm{N}=16\end{array}$ & $\mathrm{p}$ & $\begin{array}{c}\text { Clopidogrel } \\
\mathrm{N}=25\end{array}$ & $\begin{array}{c}\text { Ticagrelor } \\
\mathrm{N}=7\end{array}$ & $\mathrm{p}$ & $\begin{array}{l}\text { Clopidogrel } \\
\mathrm{N}=19\end{array}$ & $\begin{array}{c}\text { Ticagrelor } \\
\mathrm{N}=18\end{array}$ & $\mathbf{p}$ \\
\hline $\begin{array}{l}\text { Hemoglobin, } \\
\text { g/dl }\end{array}$ & $\begin{array}{c}13.4 \\
(12.3-13.9)\end{array}$ & $\begin{array}{c}12.9 \\
(12.5-14.5) \\
\end{array}$ & 0.6 & $\begin{array}{c}12.7 \\
(12.1-13.6) \\
\end{array}$ & $\begin{array}{c}12.7 \\
(11.8-13.1)\end{array}$ & 0.5 & $\begin{array}{c}12.9 \\
(11.9-14.1)\end{array}$ & $\begin{array}{c}13.4 \\
(12.8-14.7)\end{array}$ & 0.2 \\
\hline $\begin{array}{l}\mathrm{WBC} \\
, / \mathrm{mm}^{3}\end{array}$ & $\begin{array}{c}7125 \\
(6115-8225)\end{array}$ & $\begin{array}{c}8470 \\
(6580-9480)\end{array}$ & 0.03 & $\begin{array}{c}6910 \\
(5132-8530)\end{array}$ & $\begin{array}{c}5850 \\
(5207-8747) \\
\end{array}$ & 0.6 & $\begin{array}{c}7960 \\
(6630-9042)\end{array}$ & $\begin{array}{c}7245 \\
(5720-8450)\end{array}$ & 0.2 \\
\hline $\begin{array}{c}\text { Neutrophils, } \\
/ \mathrm{mm}^{3}\end{array}$ & $\begin{array}{c}4270 \\
(3505-4915) \\
\end{array}$ & $\begin{array}{c}5460 \\
(3920-6050) \\
\end{array}$ & 0.03 & $\begin{array}{c}3950 \\
(3005-5355) \\
\end{array}$ & $\begin{array}{c}3250 \\
(2942-5682) \\
\end{array}$ & 1.0 & $\begin{array}{c}4690 \\
(3737-5607) \\
\end{array}$ & $\begin{array}{c}4300 \\
(3110-5260) \\
\end{array}$ & 0.4 \\
\hline $\begin{array}{c}\text { Lymphocytes, } \\
/ \mathrm{mm}^{3}\end{array}$ & $\begin{array}{c}1880 \\
(1550-2175) \\
\end{array}$ & $\begin{array}{c}2125 \\
(1635-2735) \\
\end{array}$ & 0.1 & $\begin{array}{c}1880 \\
(1535-2172) \\
\end{array}$ & $\begin{array}{c}1800 \\
(1597-2017) \\
\end{array}$ & 0.6 & $\begin{array}{c}2000 \\
(1622-2362) \\
\end{array}$ & $\begin{array}{c}2010 \\
(1740-2760) \\
\end{array}$ & 0.4 \\
\hline $\begin{array}{c}\text { Platelet count } \\
* 10^{3} / \mu 1\end{array}$ & $\begin{array}{c}224.5 \\
(186.5-252.0) \\
\end{array}$ & $\begin{array}{c}225.0 \\
(206.5-267.0) \\
\end{array}$ & 0.2 & $\begin{array}{c}217.0 \\
(166.75-242.0)\end{array}$ & $\begin{array}{c}179.0 \\
(135.5-246.0)\end{array}$ & 0.4 & $\begin{array}{c}215.0 \\
(192.0-253.7)\end{array}$ & $\begin{array}{c}221.0 \\
(193.0-281.0) \\
\end{array}$ & 0.6 \\
\hline MPV, fL & $\begin{array}{c}10.7 \\
(10.1-11.4)\end{array}$ & $\begin{array}{c}10.8 \\
(10.0-11.7) \\
\end{array}$ & 0.7 & $\begin{array}{c}11.1 \\
(10.4-12.1)\end{array}$ & $\begin{array}{c}10.7 \\
(9.8-11.8)\end{array}$ & 0.2 & $\begin{array}{c}10.5 \\
(9.9-11.95) \\
\end{array}$ & $\begin{array}{c}10.5 \\
(9.8-11.1)\end{array}$ & 0.7 \\
\hline P-LCR, \% & $\begin{array}{c}31.7 \\
(26.7-36.6)\end{array}$ & $\begin{array}{c}31.4 \\
(25.4-39.3) \\
\end{array}$ & 0.8 & $\begin{array}{c}33.3 \\
(28.7-42-7)\end{array}$ & $\begin{array}{c}31.0 \\
(23.7-39.6)\end{array}$ & 0.2 & $\begin{array}{c}29.4 \\
(24.9-40.8) \\
\end{array}$ & $\begin{array}{c}30.1 \\
(25.5-33.7) \\
\end{array}$ & 0.9 \\
\hline PDW, fL & $\begin{array}{c}13.0 \\
(11.9-14.3) \\
\end{array}$ & $\begin{array}{c}12.8 \\
(11.6-15.1) \\
\end{array}$ & 0.8 & $\begin{array}{c}13.5 \\
(12.5-16.1) \\
\end{array}$ & $\begin{array}{c}13.1 \\
(10.7-15.2) \\
\end{array}$ & 0.2 & $\begin{array}{c}12.6 \\
(11.2-15.4) \\
\end{array}$ & $\begin{array}{c}12.0 \\
(11.4-14.3) \\
\end{array}$ & 0.6 \\
\hline
\end{tabular}


In patients undergoing percutaneous coronary intervention with stent implantation, dual antiplatelet therapy with aspirin and either clopidogrel or ticagrelor has no impact on platelet indices derived from platelet volume measurement. No conclusion can be drawn directly regarding young platelet production.

\section{REFERENCES}

1. De Luca G, Secco GG, Iorio S, et al. Short-term effects of aspirin and clopidogrel on mean platelet volume among patients with acute coronary syndromes. A single-center prospective study. Blood Coagul Fibrinolysis. 2012;23(8):756-9.

2. Vogiatzis I, Samaras A, Grigoriadis S, et al. The Mean Platelet Volume in the Prognosis of Coronary Artery Disease Severity and Risk Stratification of Acute Coronary Syndromes. Med Arch (Sarajevo, Bosnia Herzegovina). 2019;73(2):76-80.

3. Ekici B, Fahri Erkan A, Alhan A, et al. Is mean platelet volume associated with the angiographic severity of coronary artery disease? Kardiol Pol. 2013;71:832-8

4. Hossein Seyyed-Mohammadzad M, Eskandari R, Rezaei Y, et al. Prognostic value of mean platelet volume in patients undergoing elective percutaneous coronary intervention. Anatol J Cardiol. 2015; 15:25-30.

5. Goncalves SC, Labinaz M, Le May M, et al. Usefulness of Mean Platelet Volume as a Biomarker for Long-Term Outcomes after Percutaneous Coronary Intervention. Am J Cardiol. 2011;107(2):204-9.

6. Guthikonda S, Alviar CL, Vaduganathan M, et al. Role of Reticulated Platelets and Platelet Size Heterogeneity on Platelet Activity After Dual Antiplatelet Therapy With Aspirin and Clopidogrel in Patients With Stable Coronary Artery Disease. J Am Coll Cardiol. 2008;52(9):743-9.

7. Kamath S, Blann AD, Lip GYH. Platelet activation: Assessment and quantification. Eur Heart J. 2001;22(17):1561-71.

8. Beyan C, Kaptan K, Ifran A. Platelet count, mean platelet volume, platelet distribution width, and plateletcrit do not correlate with optical platelet aggregation responses in healthy volunteers. J Thromb Thrombolysis. 2006;22(3):161-4.

9. Jakl M, Sevcik R, Ceral J, et al. Mean platelet volume and platelet count: Overlooked markers of high on-treatment platelet reactivity and worse outcome in patients with acute coronary syndrome. Anadolu Kardiyol Derg. 2014;14(1):85-6.

10. Verdoia M, Pergolini P, Rolla R, et al. Mean platelet volume and high-residual platelet reactivity in patients receiving dual antiplatelet therapy with clopidogrel or ticagrelor. Expert Opin Pharmacother. 2015;16(12):173947.

11. Alvitigala BY, Azra MAF, Kottahachchi DU, et al. A study of association between platelet volume indices and ST elevation myocardial infarction. IJC Hear Vasc. 2018;21:710 . 